\title{
MAGNETIC FIELD AND ENDOSCOPE INFLUENCES ON PERISTALTIC TRANSPORT: AN EXACT SOLUTION
}

\author{
T. Hayat*1 S. Abelman, ${ }^{* 2}$ E. Momoniat* and F. M. Mahomed* \\ * School of Computational and Applied Mathematics, University of the \\ Witwatersrand, Johannesburg, Private Bag 3, Wits 2050, South Africa. \\ pensy_t@yahoo.com, Shirley.Abelman@wits.ac.za, \\ Ebrahim.Momoniat@wits.ac.za, Fazal.Mahomed@wits.ac.za
}

\begin{abstract}
The effect of magnetic field on peristaltic flow through the gap between uniform tubes is studied under the assumption of long wavelength at low Reynolds number. The inner tube is rigid and the outer tube has a sinusoidal wave travelling down its wall. The flow is investigated in a wave frame of reference moving with the velocity of the wave. The analytical solution for velocities and pressure gradient is derived. The effects of magnetic field and an endoscope on the velocities, pressure gradient, pressure rise and frictional forces on the inner and outer tubes are examined.
\end{abstract}

Keywords- Peristalsis modelling, velocity profiles, long wavelength, low Reynolds number, endoscope.

\section{INTRODUCTION}

Peristaltic transport is a form of fluid transport generated by a progressive wave of area contraction or expansion along the length of a distensible tube containing fluid. Fluid transport through muscular tubes by means of peristaltic waves is an important biological mechanism and is found in swallowing food through the esophagus, transport of urine from kidney to bladder, movement of chyme in the gastro-intestinal tract, intra-uterine fluid motion, transport of spermatozoa in the ductus efferentes of the male reproductive tract, in movement of ovum in the female fallopian tube, vasomotion of small blood vessels and in many other glandular ducts. The mechanism of peristaltic transport has been also exploited for industrial applications such as sanitary fluid transport, blood pumps in heart lung mechanics

\footnotetext{
${ }^{1}$ Permanent address: Department of Mathematics, Quaid-i-Azam University 45320, Islamabad, Pakistan and Department of Mathematics, College of Sciences, King Saud University, P. O. Box 2455, Riyadh 11451, Saudi Arabia.

${ }^{2}$ Corresponding Author
} 
and transport of corrosive fluids where the contact of the fluid with the machinery parts is prohibited. To understand peristaltic action in various situations, several theoretical and experimental investigations have been made. Important contributions to the topic on Newtonian or non-Newtonian fluids include the studies of Latham [1], Shapiro et al. [2], Fung and Yih [3], Yin and Fung [4], Shukla and Gupta [5], Srivastava and Srivastava [6], Takabatake and Ayukawa [7], Weinberg et al. [8], Yin and Fung [9], Eytan et al. [10], Srivastava and Saxena [11], Böhme and Friedrich [12], Siddiqui and Schwarz [13, 14], Hayat et al. [15, 16, 17], etc.

In all the studies mentioned above, the effect of an endoscope on the peristalsis has not been considered. More recently, Hakeem et al. [18] studied the effects of an endoscope and a generalized Newtonian fluid on the peristaltic motion. The fluid considered in reference [18] is hydrodynamic. Moreover a catheter placed within a two-dimensional model having oscillating walls has been analyzed by Rau and Usha [19], Roos and Lykoudis [20] and Yaniv et al. [21]. The influence of magnetic field on the blood flow has been discussed by Sud et al. [22]. They found that the blood speed is accelerated under the effect of a suitable moving magnetic field. Also, Agrawal and Anwaruddin [23] discussed the influence of magnetic field on blood flow by taking a simple mathematical model for blood flow through an equally branched channel with flexible walls executing peristaltic waves employing the long wavelength approximation. Mekheimer [24] studied the effect of magnetic field on peristaltic transport of blood in a non-uniform two dimensional channel, when blood is represented by a couple stress fluid. Helmy [25] obtained similarity solutions for the unsteady flow of a power-law fluid on a porous plate moving uniformly in the presence of a transverse magnetic field. An analytical solution for the velocity field and coefficient of friction of boundary layer equations for a power-law fluid in a transverse variable magnetic field is obtained by Helmy [26].

In recent years some attempts have been made to study the effects of magnetic field and an endoscope simultaneously on peristaltic motion (see [27-40] and the references mentioned there). The magnetohydrodynamic (MHD) peristaltic flow of a fluid is of interest in connection with certain problems of the movement of conductive physiological fluids e.g. the blood and blood pump machines. The purpose of this paper is to study the peristaltic flow of a magnetohydrodynamic fluid through the gap between co-axial uniform tubes. The inner tube (an endoscope) is rigid and the outer tube has a sinusoidal wave travelling down its wall. The present analysis has been carried out under the assumption of long wavelength at low Reynolds num- 
ber. This assumption is applicable since the radius $(1.25 \mathrm{~cm})$ of the small intestine is small as compared with the wavelength $(\lambda=8.01 \mathrm{~cm})$. The governing problem is solved analytically and effects of Hartmann number on the velocity components, pressure gradient, pressure rise and frictional forces on the inner and outer tubes are analyzed and discussed in detail.

The paper is organised as follows. In Section 2 the problem is formulated mathematically. In Section 3 an analytical solution to the problem is obtained. The integrals are evaluated numerically using NIntegrate in MATHEMATICA ${ }^{\circledR}$. In Section 4 the effects of magnetic field and an endoscope on the velocities, pressure gradient, pressure rise and frictional forces on the inner and outer tubes are discussed and in Section 5 conclusions are presented.

\section{FORMULATION OF THE PROBLEM}

Consider the magnetohydrodynamic flow of a viscous, incompressible and electrically conducting fluid through the gap between inner and outer tubes. The inner tube is an endoscope and the outer tube has a sinusoidal wave travelling down its wall. The surface of the tubes is electrically insulated. The geometry of the problem is shown in Fig. 1. We choose a cylindrical polar coordinate system $(\bar{R}, \bar{Z})$ with $\bar{R}$ in the radial direction and $\bar{Z}$ along the centerline of the inner and outer tubes. A uniform magnetic field $\mathbf{B}_{\mathbf{0}}$ is applied transversely to the flow. The magnetic Reynolds number is small and so the induced magnetic field is negligible. The geometry of the two wall surfaces is defined through the following equations

$$
\begin{aligned}
& \bar{r}_{1}=a_{1}, \\
& \bar{r}_{2}=a_{2}+b \sin 2 \pi(\bar{Z}-c \bar{t}),
\end{aligned}
$$

in which $a_{1}$ is the radius of the inner tube, $a_{2}$ is the radius of the outer tube at the inlet, $b$ is the amplitude of the wave (wavelength $\lambda$ ), $c$ is the propagation velocity and $\bar{t}$ is the time.

The Navier-Stokes equations and the continuity equation which govern the flow are:

$$
\begin{gathered}
\rho\left[\frac{\partial}{\partial \bar{t}}+\bar{U} \frac{\partial}{\partial \bar{R}}+\bar{W} \frac{\partial}{\partial \bar{Z}}\right] \bar{U}=-\frac{\partial \bar{p}}{\partial \bar{R}}+\mu\left(\frac{\partial^{2}}{\partial \bar{R}^{2}}+\frac{1}{\bar{R}} \frac{\partial}{\partial \bar{R}}+\frac{\partial^{2}}{\partial \bar{Z}^{2}}-\frac{1}{\bar{R}^{2}}\right) \bar{U} \\
\rho\left[\frac{\partial}{\partial \bar{t}}+\bar{U} \frac{\partial}{\partial \bar{R}}+\bar{W} \frac{\partial}{\partial \bar{Z}}\right] \bar{W}=-\frac{\partial \bar{p}}{\partial \bar{Z}}+\mu\left(\frac{\partial^{2}}{\partial \bar{R}^{2}}+\frac{1}{\bar{R}} \frac{\partial}{\partial \bar{R}}+\frac{\partial^{2}}{\partial \bar{Z}^{2}}\right) \bar{W}-\sigma B_{0}^{2} \bar{W}
\end{gathered}
$$




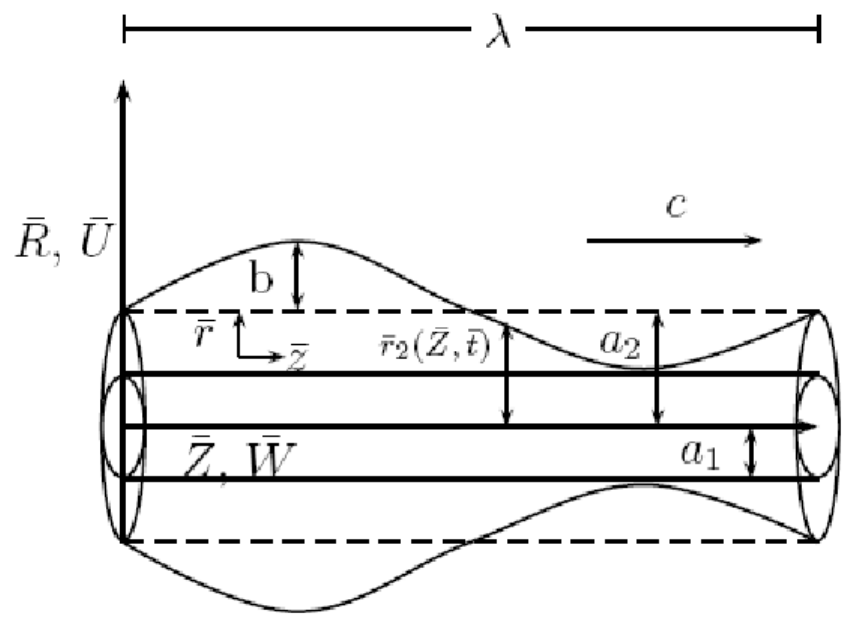

Figure 1: Effects of an endoscope on peristaltic motion of a MHD fluid.

$$
\frac{\partial \bar{U}}{\partial \bar{R}}+\frac{\bar{U}}{\bar{R}}+\frac{\partial \bar{W}}{\partial \bar{Z}}=0
$$

where $\mu$ is the dynamic viscosity, $\sigma$ is the electrical conductivity of the fluid, $\rho$ is the fluid density, $\bar{p}$ is the pressure and $\bar{U}$ and $\bar{W}$ are the velocities in laboratory frame. We shall carry out the analysis in a wave frame in which the flow is steady. The coordinates and velocities in the laboratory frame $(\bar{R}, \bar{Z})$ and the wave frame $(\bar{r}, \bar{z})$ are related through

$$
\begin{aligned}
\bar{z} & =\bar{Z}-c \bar{t}, & \bar{r} & =\bar{R}, \\
\bar{w} & =\bar{W}-c, & \bar{u} & =\bar{U},
\end{aligned}
$$

where $\bar{u}$ and $\bar{w}$ are the velocities in the wave frame. The boundary conditions in the wave frame are [18]

$$
\begin{aligned}
& \bar{w}=-c \text { at } \bar{r}=\bar{r}_{1}, \quad \bar{r}=\bar{r}_{2} \\
& \bar{u}=0 \text { at } \bar{r}=\bar{r}_{1} .
\end{aligned}
$$

Employing the transformations (6) and (7) and then defining the dimensionless variables

$$
r=\bar{r} / a_{2}, \quad r_{1}=\bar{r}_{1} / a_{2}=a_{1} / a_{2}=\epsilon<1, \quad z=\bar{z} / a_{2},
$$




$$
w=\bar{w} / c, \quad u=\lambda \bar{u} / a_{2} c, \quad p=a_{2}^{2} \bar{p} / c \lambda \mu, \quad t=c \bar{t} / \lambda,
$$

Eqs. (3)-(5) and boundary conditions (8)-(9) become

$$
\begin{aligned}
\operatorname{Re} \delta^{3}\left[u \frac{\partial}{\partial r}+w \frac{\partial}{\partial z}\right] u= & -\frac{\partial p}{\partial r}+2 \delta^{2} \frac{\partial^{2} u}{\partial r^{2}}+\delta^{2} \frac{\partial}{\partial z}\left(\frac{\partial w}{\partial r}+\delta^{2} \frac{\partial u}{\partial z}\right) \\
& +\frac{2 \delta^{2}}{r}\left(\frac{\partial u}{\partial r}-\frac{u}{r}\right), \\
\operatorname{Re} \delta\left[u \frac{\partial}{\partial r}+w \frac{\partial}{\partial z}\right] w= & -\frac{\partial p}{\partial z}+\frac{1}{r} \frac{\partial}{\partial r}\left[r\left(\frac{\partial w}{\partial r}+\delta^{2} \frac{\partial u}{\partial z}\right)\right] \\
& +2 \delta^{2} \frac{\partial^{2} w}{\partial z^{2}}-M^{2}(w+1), \\
= & \frac{\partial u}{\partial r}+\frac{u}{r}+\frac{\partial w}{\partial z}=0, \\
w=0 \quad \text { at } r=r_{1}=\epsilon, & \quad r=r_{2}, \\
r_{2}=1+\phi \sin 2 \pi z, &
\end{aligned}
$$

where the dimensionless wave number $(\delta)$, Reynolds number (Re), the amplitude ratio $(\phi)$ and Hartmann number $(\mathrm{M})$ are respectively given by

$$
\begin{aligned}
\delta & =\frac{a_{2}}{\lambda} \ll 1, \quad R e=\frac{\rho c a_{2}}{\mu}, \quad \phi=b / a_{2}<1, \\
M & =\sqrt{\frac{\sigma}{\mu}} B_{0} a_{2}>\sqrt{2}
\end{aligned}
$$

and $\epsilon$ is the radius ratio. The Eqs. (11)-(12) for long wavelength with low Reynolds number approximation become

$$
\begin{aligned}
0 & =-\frac{\partial p}{\partial r} \\
\frac{\partial p}{\partial z} & =\frac{1}{r} \frac{\partial}{\partial r}\left[r \frac{\partial w}{\partial r}\right]-M^{2}(w+1)
\end{aligned}
$$

where Eq. (18) shows that $p$ is not a function of $r$. Hence $p$ is only a function of $z$.

In dimensionless variables the expressions for volume flow rate $(\mathrm{F})$, pressure rise $\left(\Delta P_{\lambda}\right)$ and frictional forces on the inner $\left(F_{\lambda}^{(\mathrm{i})}\right)$ and outer tubes $\left(F_{\lambda}^{(\mathrm{o})}\right)$ are 
respectively given by [18]

$$
\begin{aligned}
F & =\int_{r_{1}}^{r_{2}} r w d r \\
\Delta P_{\lambda} & =\int_{0}^{1}\left(\frac{d p}{d z}\right) d z \\
F_{\lambda}^{(\mathrm{i})} & =\int_{0}^{1} r_{1}^{2}\left(-\frac{d p}{d z}\right) d z \\
F_{\lambda}^{(\mathrm{o})} & =\int_{0}^{1} r_{2}^{2}\left(-\frac{d p}{d z}\right) d z .
\end{aligned}
$$

\section{ANALYTICAL SOLUTION}

Since the right-hand side of Eq. (19) is a function of $z$ only, we have that

$$
\begin{aligned}
& \frac{d p}{d z}=\lambda_{1}(z), \\
& \frac{1}{r} \frac{d}{d r}\left[r \frac{d w}{d r}\right]-M^{2}(w+1)=\lambda_{1}(z) .
\end{aligned}
$$

Eq. (25) can easily be solved to give

$$
w(r)=A_{1} I_{0}(M r)+A_{2} K_{0}(M r)-\left(\lambda_{1}(z)+M^{2}\right) / M^{2},
$$

where $I_{0}$ is the modified Bessel function of the first kind of order $0, K_{0}$ is the modified Bessel function of the second kind of order 0 and $A_{1}$ and $A_{2}$ are constants to be determined after imposing the boundary conditions (14). Imposing the boundary conditions on Eq. (26) we find that

$$
\begin{aligned}
& A_{1}=\frac{\lambda_{1}(z)\left(K_{0}(\epsilon M)-K_{0}\left(M r_{2}\right)\right)}{M^{2}\left(I_{0}\left(M r_{2}\right) K_{0}(\epsilon M)-I_{0}(\epsilon M) K_{0}\left(M r_{2}\right)\right)}, \\
& A_{2}=\frac{\lambda_{1}(z)\left(I_{0}\left(M r_{2}\right)-I_{0}(\epsilon M)\right)}{M^{2}\left(I_{0}\left(M r_{2}\right) K_{0}(\epsilon M)-I_{0}(\epsilon M) K_{0}\left(M r_{2}\right)\right)} .
\end{aligned}
$$

We can calculate $\lambda_{1}(z)$ in terms of the flow rate $F$ through Eq. (20). Taking $r_{1}=\epsilon$ we find that

$$
\begin{gathered}
\frac{d p}{d z}=\lambda_{1}(z)=\left[M^{4}\left(I_{0}\left(M r_{2}(z)\right) K_{0}(\epsilon M)-I_{0}(\epsilon M) K_{0}\left(M r_{2}(z)\right)\right)\right. \\
\left.\left(\epsilon^{2}-2 F-r_{2}(z)^{2}\right)\right] \times\left[4+\epsilon^{2} M^{2}\left(I_{2}(\epsilon M) K_{0}\left(M r_{2}(z)\right)\right.\right. \\
\left.-I_{0}(M r 2(z)) K_{2}(\epsilon M)\right)+M r_{2}(z)\left(-2\left(I_{1}\left(M r_{2}(z)\right) K_{0}(\epsilon M)\right.\right. \\
\left.+I_{0}(\epsilon M) K_{1}\left(M r_{2}(z)\right)\right)+M\left(I_{0}\left(M r_{2}(z)\right) K_{0}(\epsilon M)\right. \\
\left.\left.\left.-I_{0}(\epsilon M) K_{0}\left(M r_{2}(z)\right)\right) r_{2}(z)\right)\right]^{-1}
\end{gathered}
$$


We plot Eq.(29) in Figs. 2-3.

We can calculate $u$ from Eq. (13) after using Eq. (26) and obtain

$$
\begin{gathered}
u=\left[2 M^{4} r\left(I_{0}\left(M r_{2}(z)\right) K_{0}(\epsilon M)-I_{0}(\epsilon M) K_{0}\left(M r_{2}(z)\right)\right)^{2} r_{2}(z)\right]^{-1} \\
{\left[\left(I_{0}\left(M r_{2}(z)\right) K_{0}(\epsilon M)-I_{0}(\epsilon M) K_{0}\left(M r_{2}(z)\right)\right)\right.} \\
\left(2+M\left(M\left(-\left(r^{2} I_{0}(\epsilon M)\right)+\epsilon^{2} I_{2}(\epsilon M)\right) K_{0}(M r 2(z))\right.\right. \\
+2 r I_{1}(M r)\left(-K_{0}(\epsilon M)+K_{0}\left(M r_{2}(z)\right)\right) \\
-2 r I_{0}(\epsilon M) K_{1}(M r)+I_{0}\left(M r_{2}(z)\right)\left(M r^{2} K_{0}(\epsilon M)+2 r K_{1}(M r)\right. \\
\left.\left.-\epsilon^{2} M K_{2}(\epsilon M)\right)\right) r_{2}(z) \lambda_{1}{ }^{\prime}(z)+2\left(-1+M r I_{1}(M r) K_{0}(\epsilon M)\right. \\
\left.+M r I_{0}(\epsilon M) K_{1}(M r)\right) \lambda_{1}(z)\left(-1+M\left(I_{1}\left(M r_{2}(z)\right) K_{0}(\epsilon M)\right.\right. \\
\left.\left.\left.+I_{0}(\epsilon M) K_{1}\left(M r_{2}(z)\right)\right) r_{2}(z)\right) r_{2}{ }^{\prime}(z)\right],
\end{gathered}
$$

where $^{\prime}=d / d z$. We plot the values of Eqs. (21)-(23) in Figs. 13-15, respectively. The values are calculated by evaluating the integrals numerically using NIntegrate in MATHEMATICA ${ }^{\circledR}$.

\section{DISCUSSION}

The effects of the Hartmann number on the pressure gradient $d p / d z$ are plotted in Figs. 2-4. The effect on $d p / d z$ under varying amplitude ratios $\phi$ is plotted in Fig. 2. From Fig. 2 we observe that the Hartmann number changes the maximum amplitude of $d p / d z$ when compared to the case with zero Hartmann number. This change in amplitude is increased by increasing the amplitude ratio $\phi$. The effect of changing flow rate on $d p / d z$ is indicated in Fig. 3. Again, we observe that there is a definite increase in the maximum amplitude of $d p / d z$ when increasing the magnitude of the flow rate when compared to the case of zero Hartmann number. In Fig. 4 we plot the change in $d p / d z$ when changing $\epsilon$. Again, there is an increase in the maximum amplitude of $d p / d z$ with increasing $\epsilon$.

In general, we can conclude that the presence of a magnetic filed increases the maximum amplitude of the pressure gradient. This increase is further compounded by increasing the amplitude ratio, $\phi$, the magnitude of the flow rate, $F$ and $\epsilon$. 

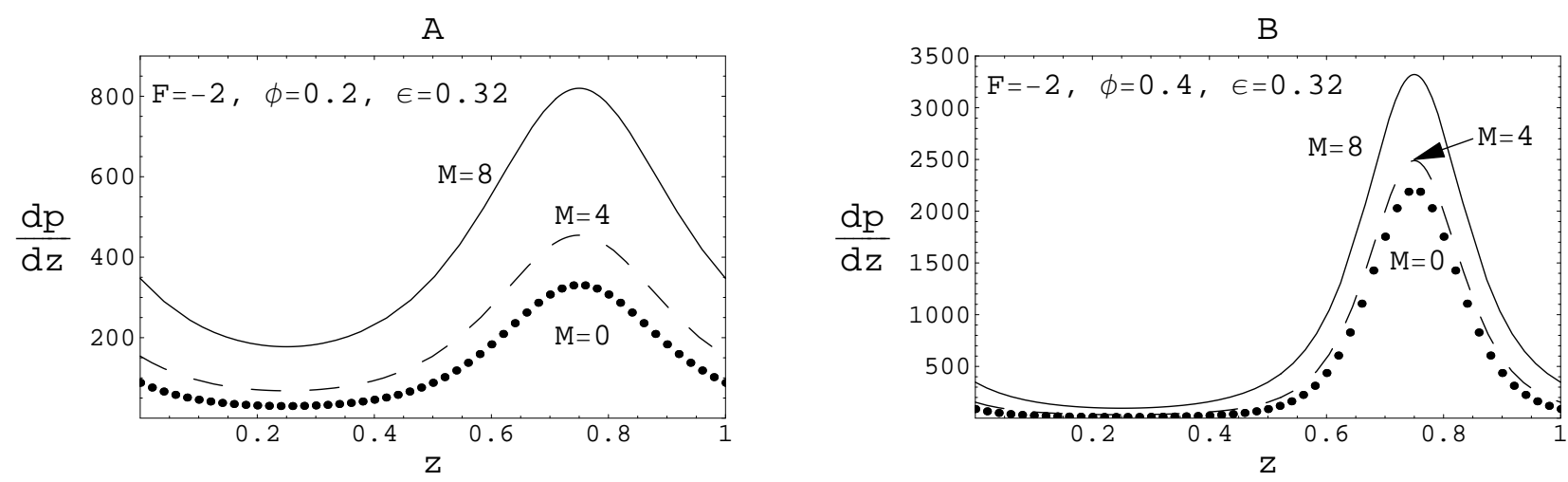

Figure 2: Plot showing variation of the pressure gradient $d p / d z$ within a wavelength $z \in[0,1]$ for different values of the Hartmann number $M$ for the amplitude ratios (A) $\phi=0.2$ and (B) $\phi=0.4$ subject to a fixed flow rate $F=-2$. We have chosen $\epsilon=0.32$.
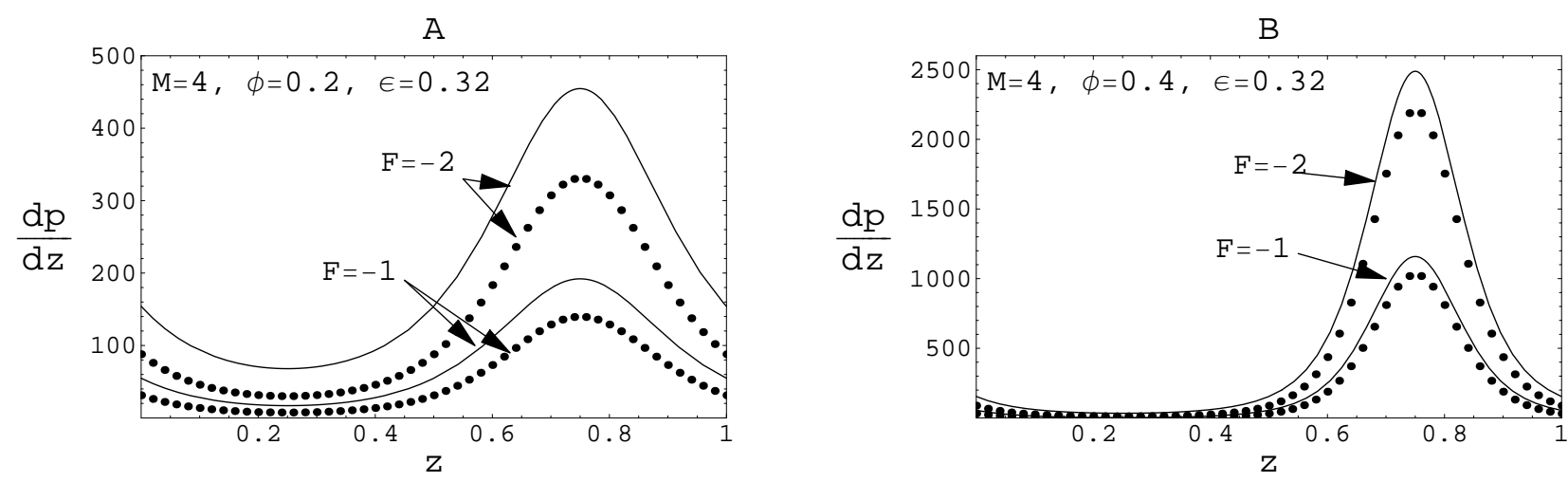

Figure 3: Plot showing variation of the pressure gradient $d p / d z$ within a wavelength $z \in[0,1]$ for a fixed Hartmann number $M=4$ for the flow rates (A) $F=-1$ and (B) $F=-2$ subject to a fixed amplitude ratio $\phi=0.2$. We have chosen $\epsilon=0.32$. The dotted lines $(\bullet \bullet \bullet)$ correspond to the case $M=0$.

The effects of the Hartmann number on the velocities $u$ and $w$ are investigated in Figs. 5-13. In Fig. 5 we plot the velocities $u(r, z)$ and $w(r, z)$ for zero Hartmann number. We have used a contour map to plot the effect of the Hartmann number on the velocities. The lighter shaded regions have a higher velocity than the regions shaded darker. Fig. 5A indicates a sink for $w$ while Fig. 5B indicates that $u$ is very sinusoidal flattening in the region for small $z$. In Figs. 6-9 the effects of changing Hartmann number, $M$, amplitude ratio $\phi$, flow rate $F$ and $\epsilon$ on $w(r, z)$ are plotted. From these figures we note that the main effect of increasing the magnitudes of $M$, $F, \phi$ and $\epsilon$ is to steepen the gradient of the sink. Also, the magnitude and width of 


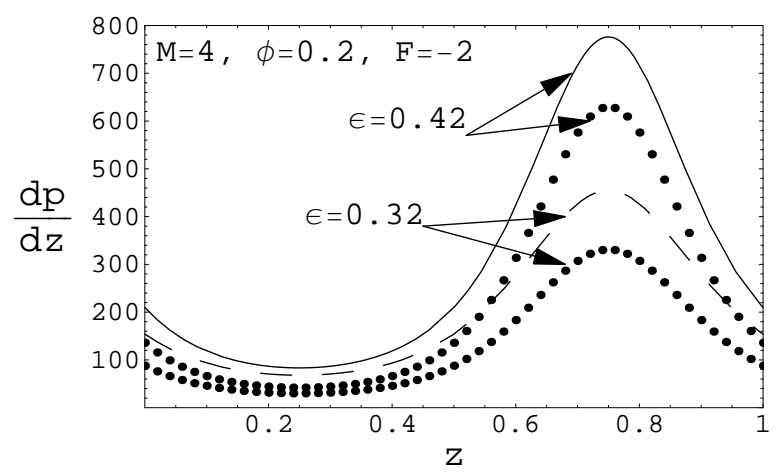

Figure 4: Plot showing variation of the pressure gradient $d p / d z$ within a wavelength $z \in[0,1]$ for a fixed Hartmann number $M=4$, flow rate $F=-2$ and amplitude ratio $\phi=0.2$ with varying $\epsilon$. The dotted lines $(\bullet \bullet \bullet)$ correspond to the case $M=0$.

the sink increases. We note that the width of the sink in Figs. 6B-9B is wider when compared with Figs. 6A-9A.
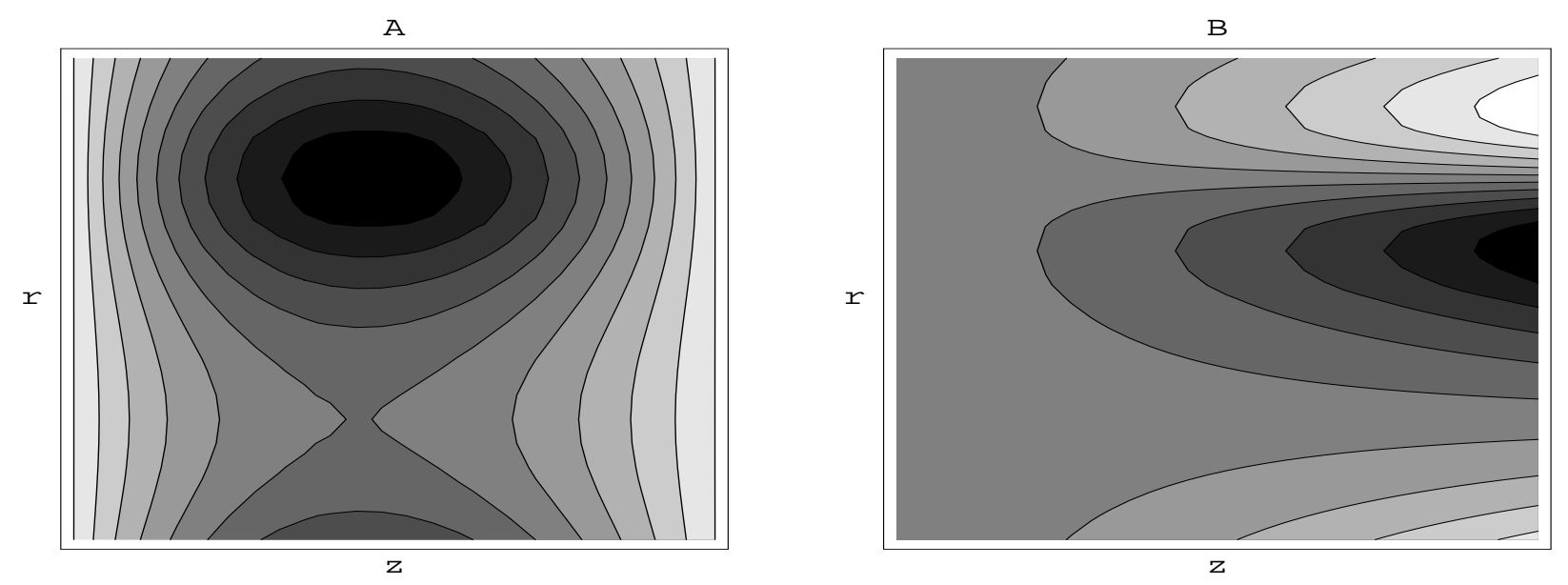

Figure 5: Plot showing $w(r, z)(\mathrm{A})$ and $u(r, z)(\mathrm{B})$ for zero Hartmann number $(M=$ $0)$ where $\epsilon=0.32, F=-2, \phi=0.2, r \in\left[\epsilon, r_{2}(z)\right]$ and $z \in[0,1]$. 
A

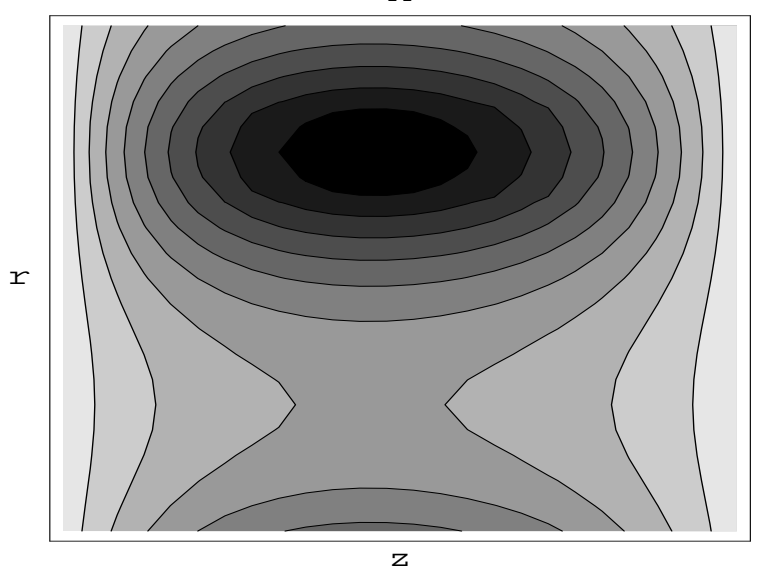

B

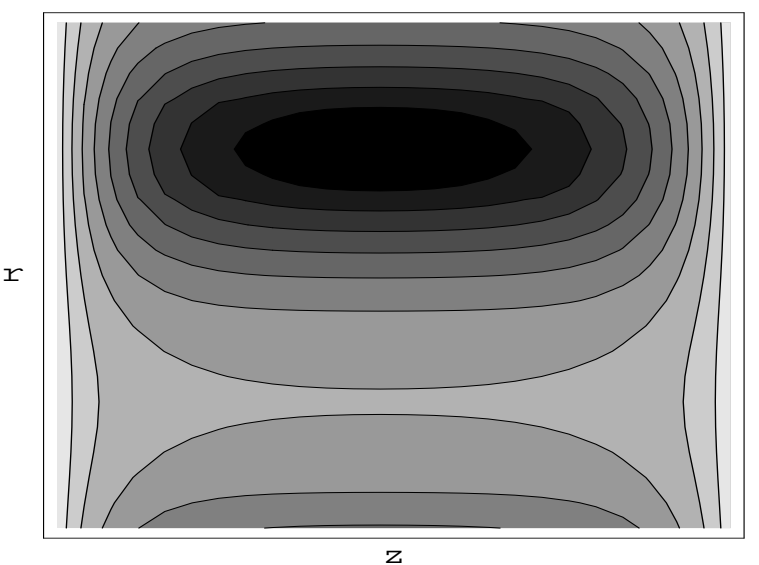

Figure 6: Plot showing $w(r, z)$ for fixed amplitude ratio $\phi=0.2$ and changing Hartmann number (A) $M=4$ (B) $M=16$, where $\epsilon=0.32$ and $F=-2, r \in\left[\epsilon, r_{2}(z)\right]$ and $z \in[0,1]$.
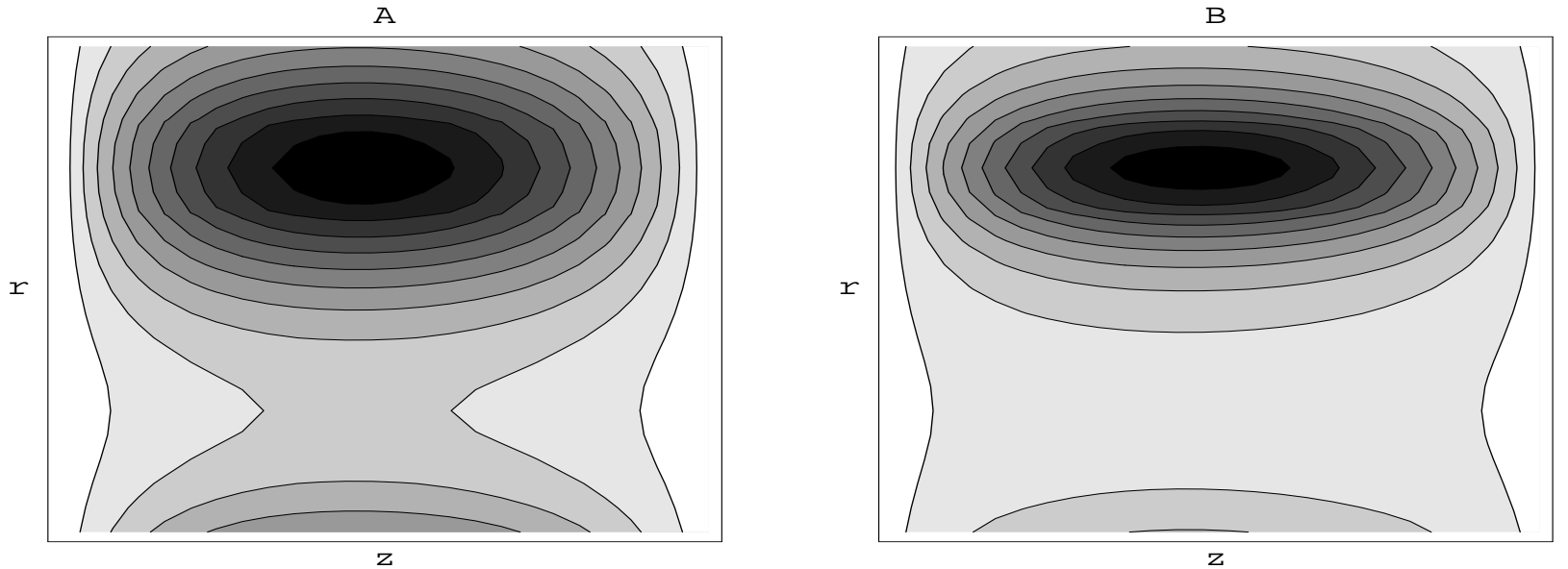

Figure 7: Plot showing $w(r, z)$ for fixed Hartmann number $M=4$ and changing flow rate and changing amplitude ratio (A) $\phi=0.2, F=-1$ (B) $\phi=0.4, F=-2$ where $\epsilon=0.32, r \in\left[\epsilon, r_{2}(z)\right]$ and $z \in[0,1]$. 
A

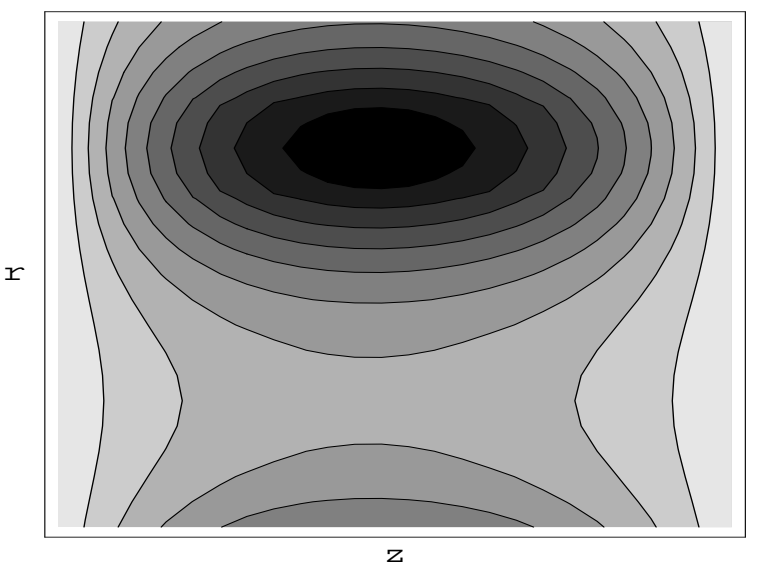

B

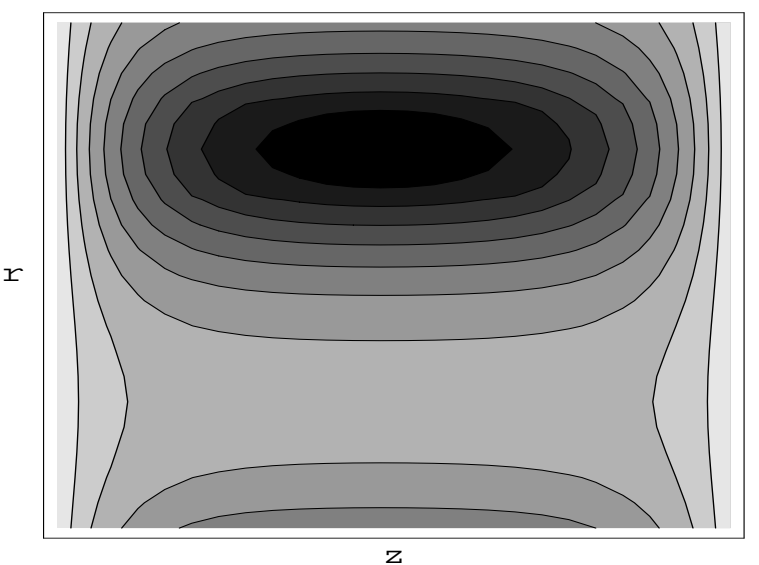

Figure 8: Plot showing $w(r, z)$ for fixed amplitude ratio $\phi=0.2$ and changing Hartmann number (A) $M=4$ (B) $M=16$, where $\epsilon=0.42$ and $F=-2, r \in\left[\epsilon, r_{2}(z)\right]$ and $z \in[0,1]$.
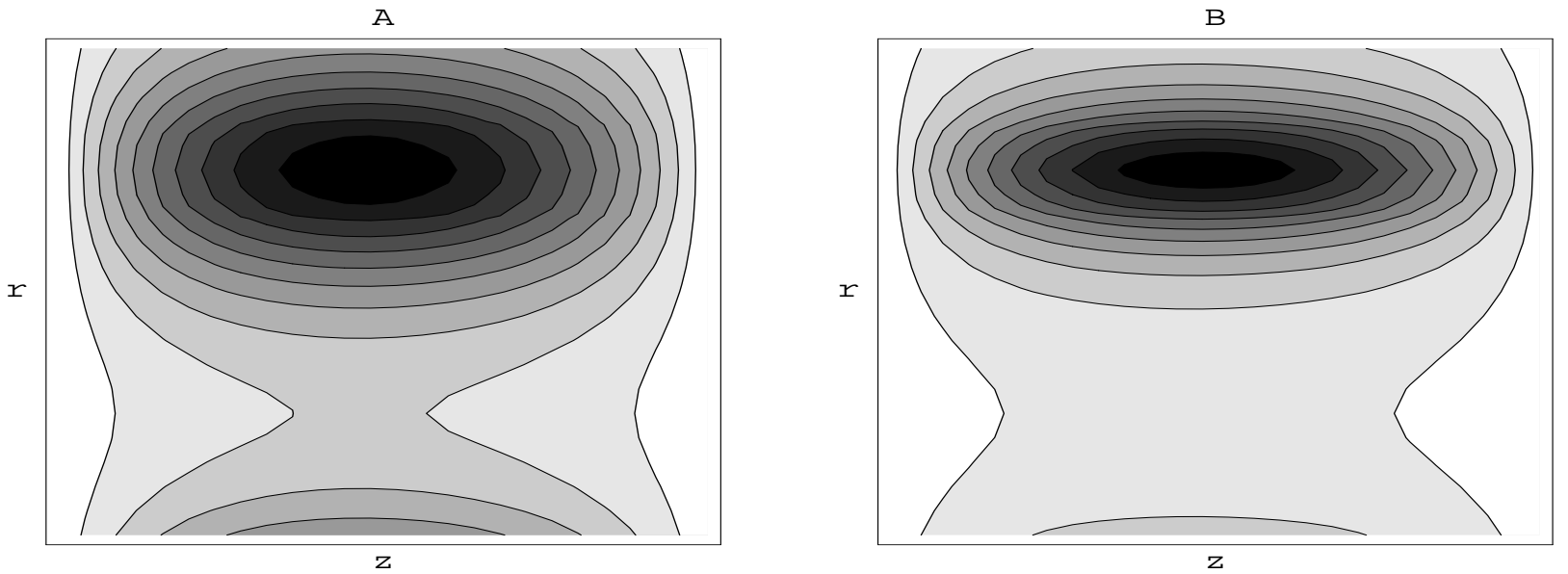

Figure 9: Plot showing $w(r, z)$ for fixed Hartmann number $M=4$ and changing flow rate and changing amplitude ratio $(\mathrm{A}) \phi=0.2, F=-1$ (B) $\phi=0.4, F=-2$ where $\epsilon=0.42, r \in\left[\epsilon, r_{2}(z)\right]$ and $z \in[0,1]$.

In Figs. 10-13 the effects of changing Hartmann number, $M$, amplitude ratio $\phi$, flow rate $F$ and $\epsilon$ on $u(r, z)$ are plotted. Here, once again, we note a steepening of the edges in the sinusoidal behaviour of $u$. There is an increase in the magnitude (depth and height of sinusoidal wave) as the parameter values increase. The change in behaviour mimics the change in behaviour indicated for $u$. 
A

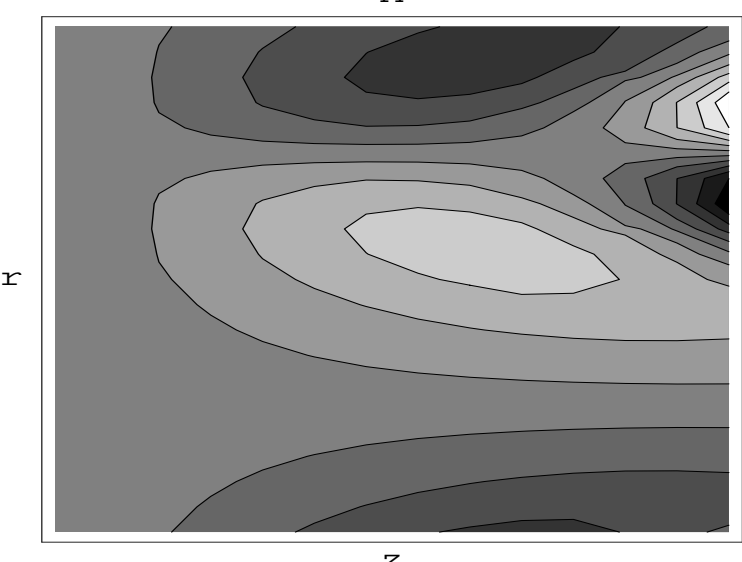

B

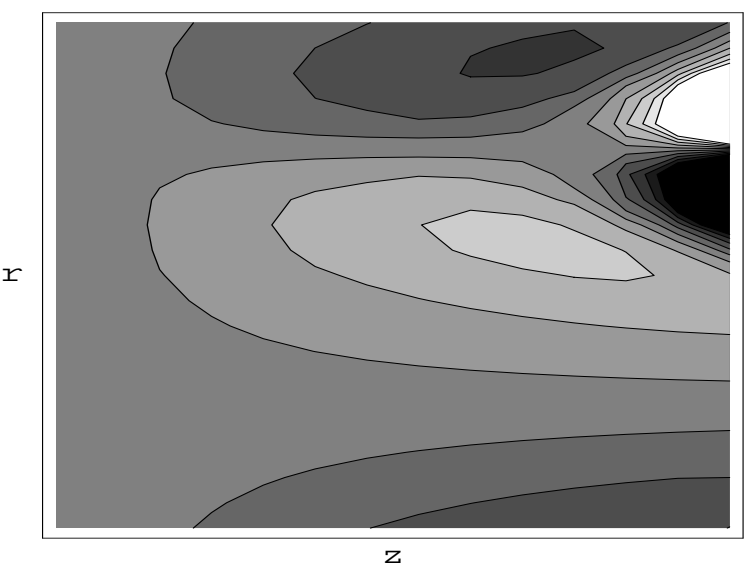

Figure 10: Plot showing $u(r, z)$ for fixed amplitude ratio $\phi=0.2$ and changing Hartmann number (A) $M=4$ (B) $M=16$, where $\epsilon=0.32, F=-2, r \in\left[\epsilon, r_{2}(z)\right]$ and $z \in[0,1]$.

A

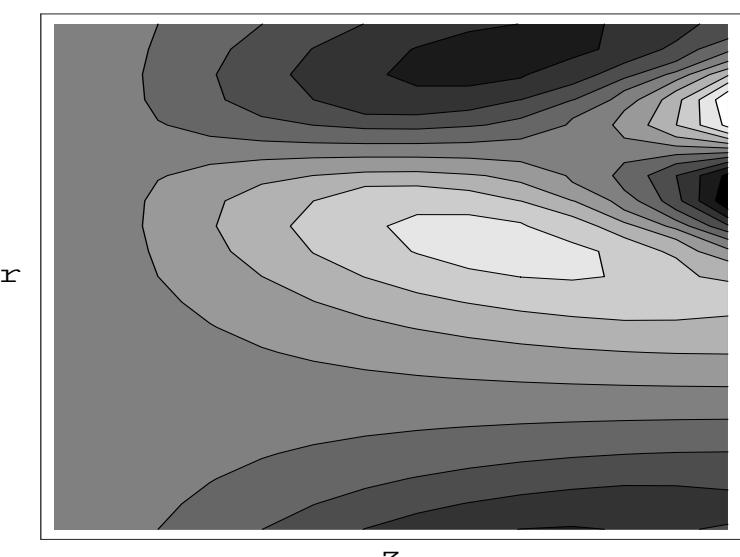

B

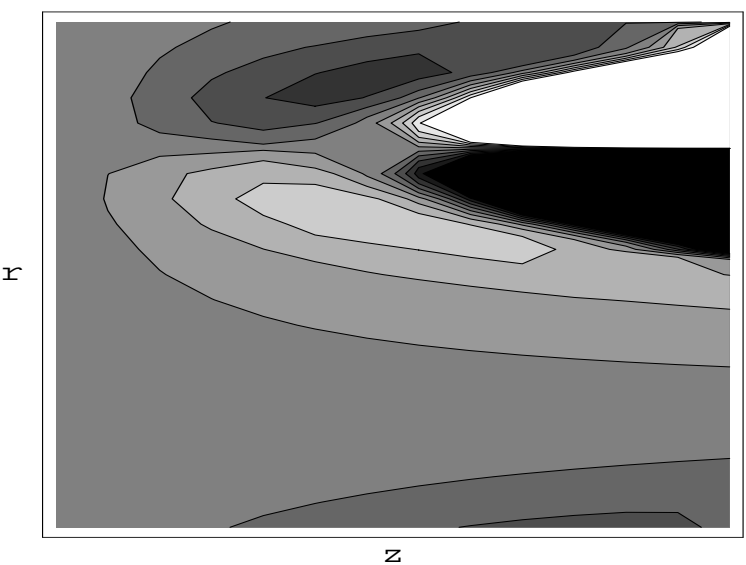

Figure 11: Plot showing $u(r, z)$ for fixed Hartmann number $M=4$ and changing flow rate and changing amplitude ratio (A) $\phi=0.2, F=-1$ (B) $\phi=0.4, F=-2$ where $\epsilon=0.32, r \in\left[\epsilon, r_{2}(z)\right]$ and $z \in[0,1]$. 
A

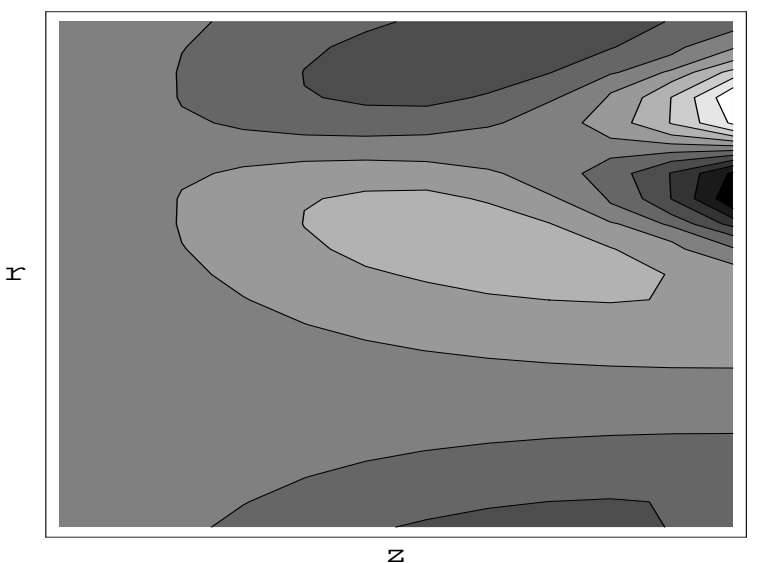

B



Figure 12: Plot showing $u(r, z)$ for fixed amplitude ratio $\phi=0.2$ and changing Hartmann number (A) $M=4$ (B) $M=16$, where $\epsilon=0.42, F=-2, r \in\left[\epsilon, r_{2}(z)\right]$ and $z \in[0,1]$.

A

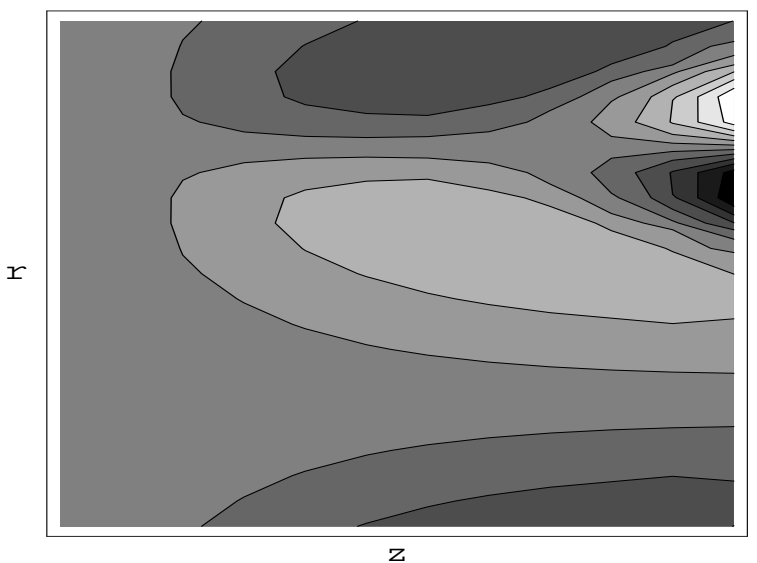

B

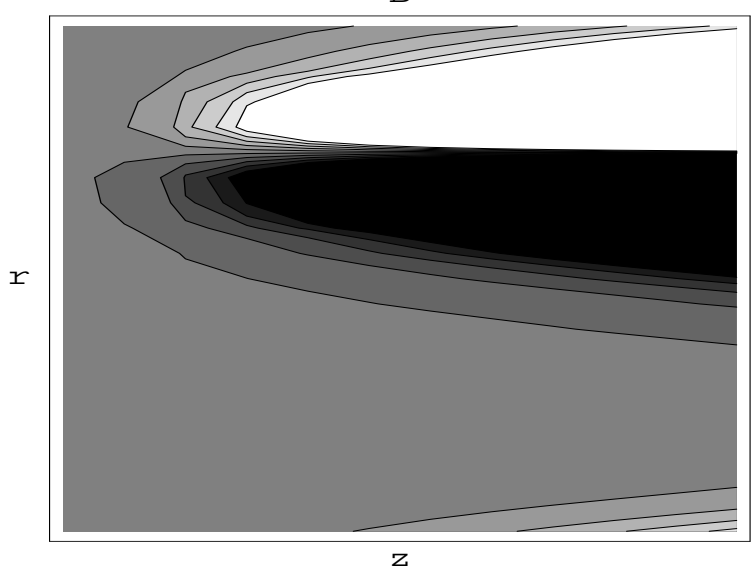

Figure 13: Plot showing $u(r, z)$ for fixed Hartmann number $M=4$ and changing flow rate and changing amplitude ratio $(\mathrm{A}) \phi=0.2, F=-1$ (B) $\phi=0.4, F=-2$ where $\epsilon=0.42, r \in\left[\epsilon, r_{2}(z)\right]$ and $z \in[0,1]$.

In Fig. 14 the effects of changing Hartmann number, $M$, amplitude ratio $\phi$, flow rate $F$ and $\epsilon$ on the pressure rise $\Delta P_{\lambda}$ are plotted. We observe that as the Hartmann number increases there is a nonlinear increase in the pressure rise. Increasing the values of the parameters $\phi, F$ and $\epsilon$ contributes to the increase in pressure rise. 

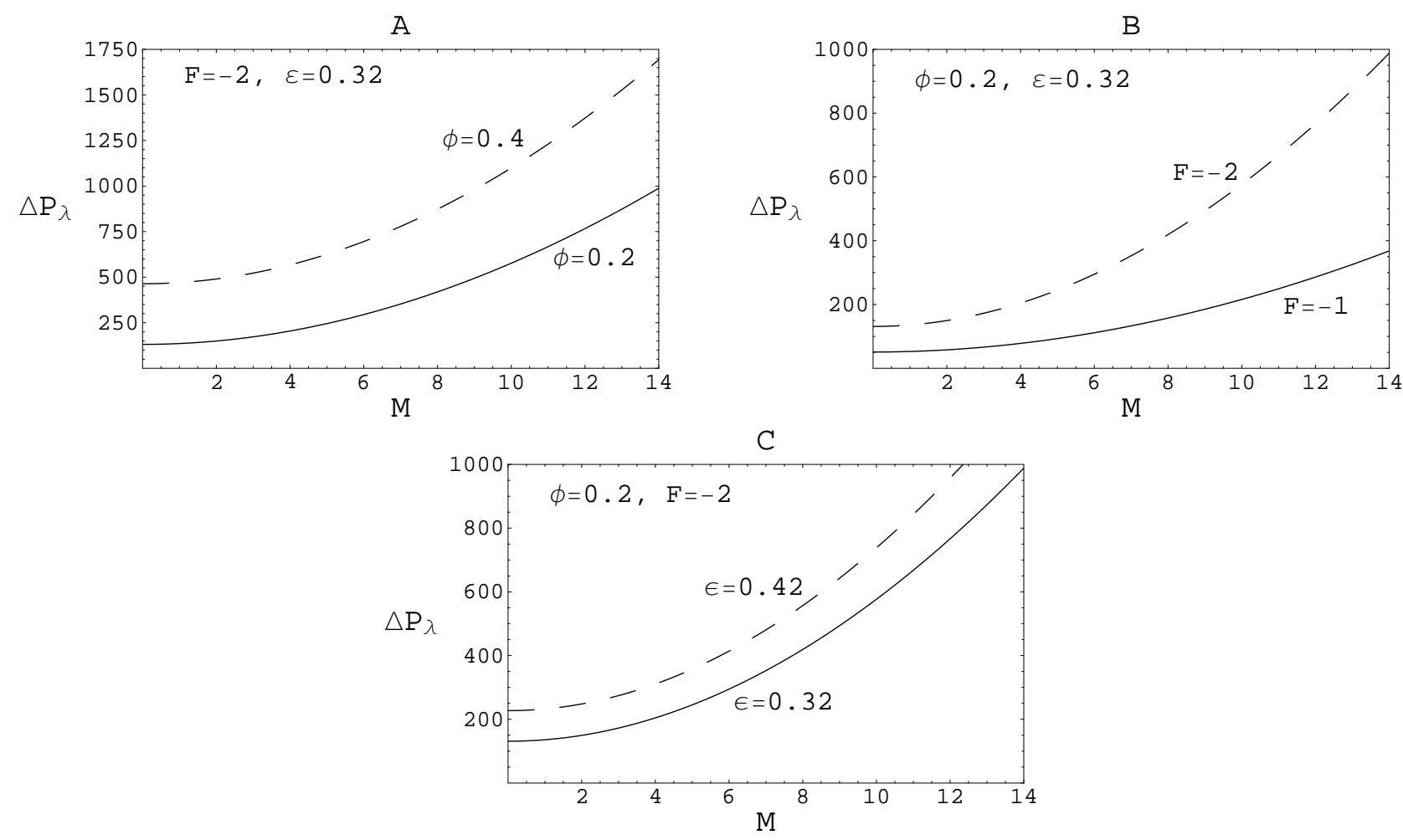

Figure 14: Plot showing $\Delta P_{\lambda}$ for $M \in[0.01,14]$ for changing amplitude ratio (A) $\phi=0.2$ and $\phi=0.4$, changing flow rate (B) $F=-1$ and $F=-2$ and changing $\epsilon$ (C) $\epsilon=0.32$ and $\epsilon=0.42$.

In Fig. 15 the effects of changing Hartmann number, $M$, amplitude ratio $\phi$, flow rate $F$ and $\epsilon$ on frictional forces on the inner tube $F_{\lambda}^{(\mathrm{i})}$ are plotted. In Fig. 16 the effects of changing Hartmann number, $M$, amplitude ratio $\phi$, flow rate $F$ and $\epsilon$ on frictional forces on the outer tube $F_{\lambda}^{(\mathrm{o})}$ are plotted. In both cases as the magnetic field increases we note an increase in the magnitude but in the opposite direction to the pressure rise. Increasing the values of the parameters $\phi, F$ and $\epsilon$ contributes to an increase in the frictional forces on the inner and outer tubes. 
652 Magnetic Field and Endoscope Influences on Peristaltic Transport
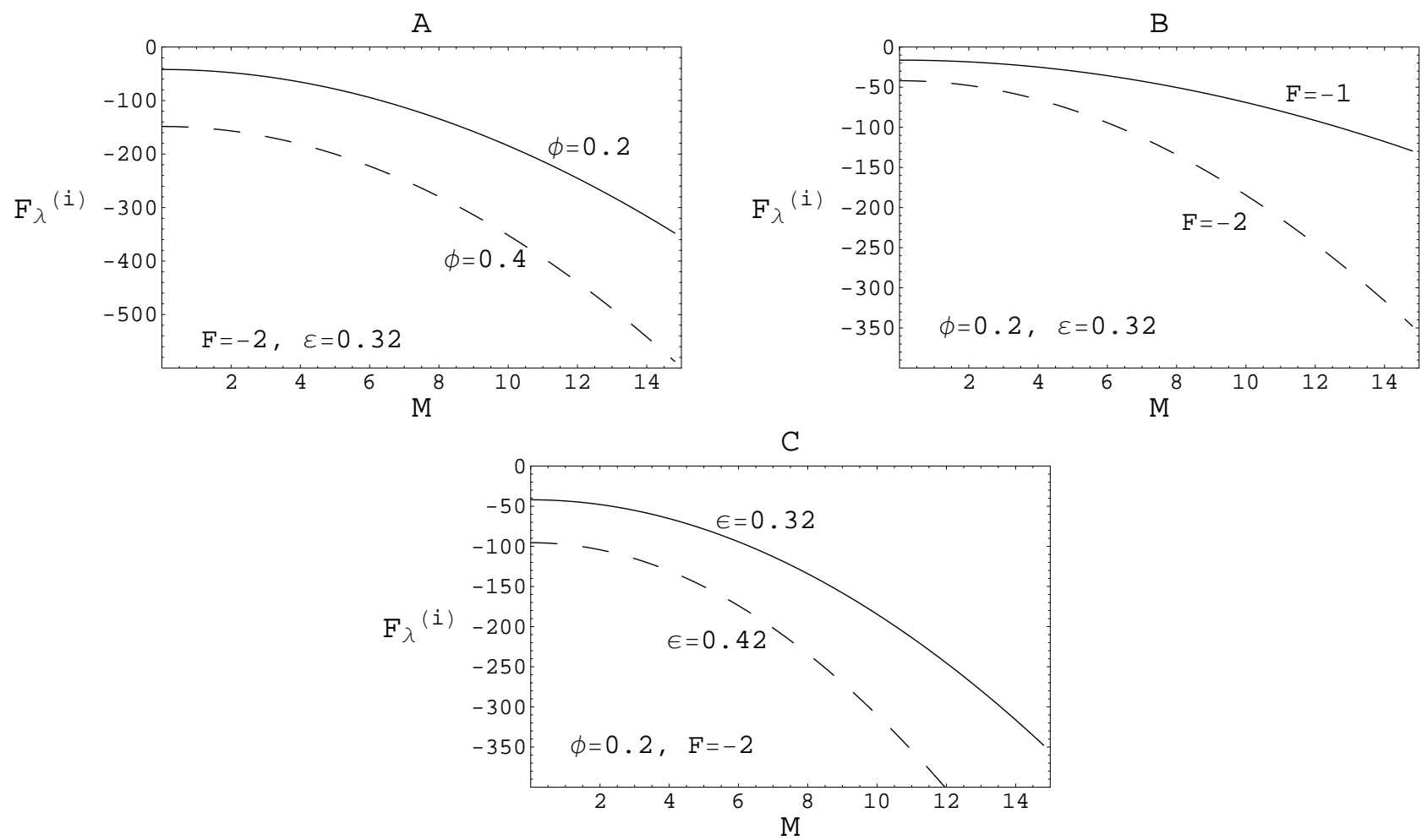

Figure 15: Plot showing $F_{\lambda}^{(\mathrm{i})}$ for $M \in[0.01,15]$ for changing amplitude ratio (A) $\phi=0.2$ and $\phi=0.4$, changing flow rate (B) $F=-1$ and $F=-2$ and changing $\epsilon$ (C) $\epsilon=0.32$ and $\epsilon=0.42$. 
A

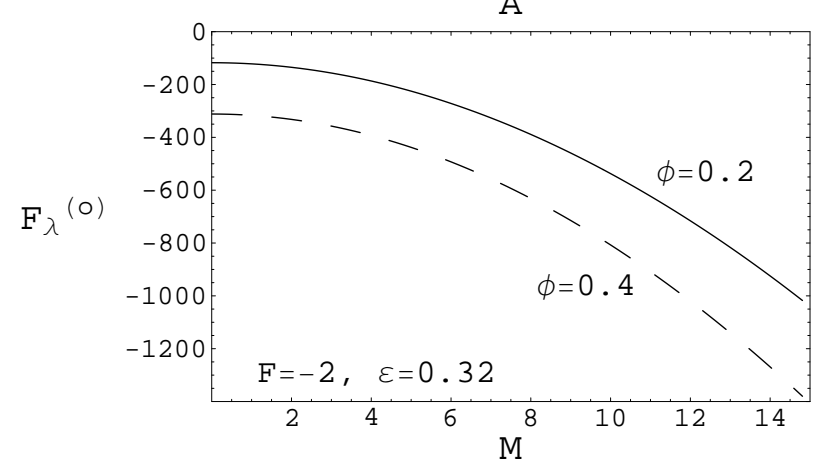

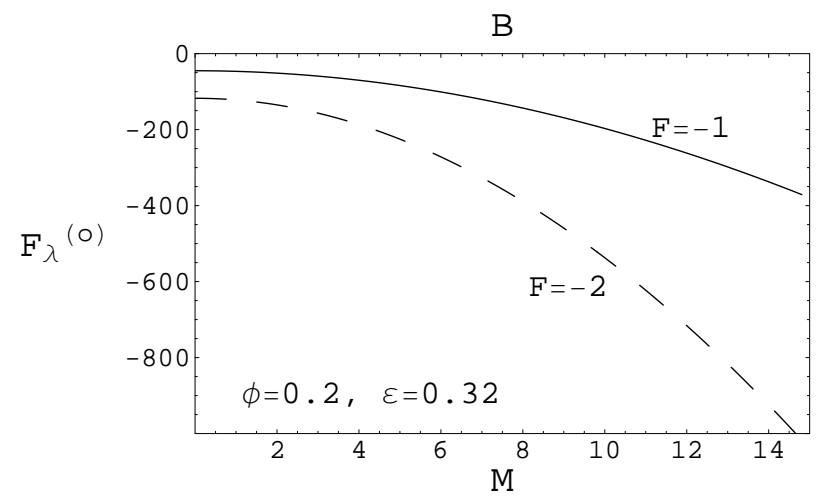

C

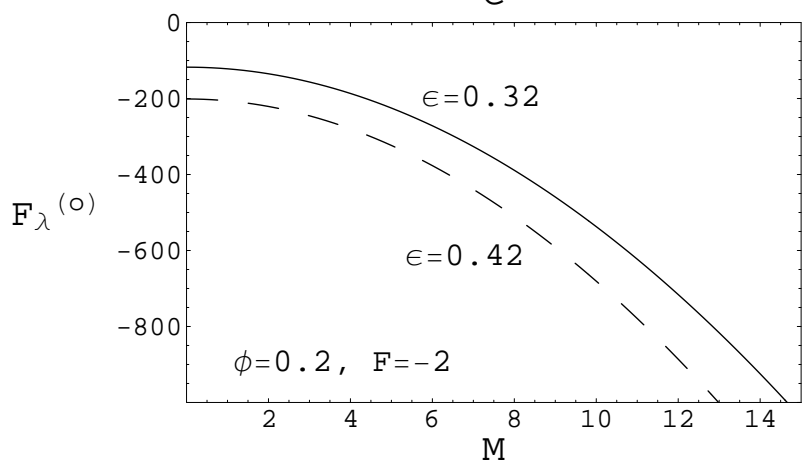

Figure 16: Plot showing $F_{\lambda}^{(0)}$ for $M \in[0.01,15]$ for changing amplitude ratio (A) $\phi=0.2$ and $\phi=0.4$, changing flow rate (B) $F=-1$ and $F=-2$ and changing $\epsilon$ (C) $\epsilon=0.32$ and $\epsilon=0.42$.

\section{CONCLUSIONS}

A mathematical model to study the peristaltic transport of magnetohydronamic (MHD) fluid along with an endoscope effect is presented. The most important characteristics of peristaltic mechanism such as the velocity components, pressure gradient (axial), pressure rise and frictional forces are discussed with the variation in Hartmann number, the amplitude ratio, radius ratio and flow rate. The graphs of analytical solutions for velocities and pressure gradient; and numerical evaluations of pressure rise and frictional forces reveal several facts. The amplitude of pressure gradient is found to be always larger in the case of an MHD fluid than in a hydrodynamic fluid. As expected, increase in Hartmann number increases pressure rise. The behaviour of frictional forces is opposite when compared to the pressure rise. Further, the pressure rise increases with increasing values of the amplitude and radius ratios. An increase in flow rate increases the pressure rise; thus maximum flow rate is achieved for large pressure rise and maximum pressure rise is achieved 
at large flow rate. The Hartmann number is also found to decrease flow velocities.

Acknowledgements- TH thanks the DECMA research centre and the School of Computational and Applied Mathematics at the University of the Witwatersrand, Johannesburg, for financial support and hospitality. EM and SA acknowledge support received from the National Research Foundation of South Africa under grant numbers 2053745 and 72518 respectively. The authors thank anonymous referees for their comments which have improved this paper.

\section{REFERENCES}

[1] T. W. Latham, Fluid motion in peristaltic pump, MS. Thesis, MIT, Cambridge, Mass, 1966.

[2] A. H. Shapiro, M. Y. Jaffrin and S. L. Weinberg, Peristaltic pumping with long wavelength at low Reynolds number, J. Fluid Mech. 37, $799-825,1969$.

[3] Y. C. Fung and C. S. Yih, Peristaltic transport, J. Appl. Mech. 35, $669-675,1968$.

[4] C. C. Yin and Y. C. Fung, Peristaltic waves in circular cylindrical tubes, J. Appl. Mech. 36, 579 - 587, 1969.

[5] J. B. Shukla and S. P. Gupta, Peristaltic transport of a power law fluid with variable consistency, J. Biomech. Eng. 104, 182 - 186, 1982.

[6] L. M. Srivastava and V. P. Srivastava, Peristaltic transport of blood: Casson model II, J. Biomech. 17, 821 - 829, 1984.

[7] S. Takabatake and K. Ayukawa, Numerical study of two-dimensional peristaltic flows, J. Fluid Mech. 122, 439 - 465, 1982.

[8] S. L. Weinberg, E. C. Eckistein and A. H. Shapiro, An experimental study of peristaltic pumping, J. Fluid. Mech. 49, 461 - 197, 1971.

[9] C. C. Yin and Y. C. Fung, Comparison of theory and experiment in peristaltic transport, J. Fluid Mech. 47, 93 - 112, 1971.

[10] O. Eytan, A. J. Jaffa, J. Har-Toov, E. Dalach and D. Elad, Dynamics of the intraterine fluid-wall interface, Annals of Biomed. Eng. 27, $372-379,1999$. 
[11] V. P. Srivastava and M. Saxena, A two-fluid model of non-Newtonian blood flow induced by peristaltic waves, Rheol. Acta. 34, 406 - 414, 1995.

[12] G. Böhme and R. Friedrich, Peristaltic flow of viscoelastic liquids, J. Fluid Mech. 128, 109 - 122, 1983.

[13] A. M. Siddiqui and W. H. Schwarz, Peristaltic flow of a second order fluid in tubes, J. Non-Newtonian Fluid Mech. 53, 257 - 284, 1994.

[14] A. M. Siddiqui and W. H. Schwarz, Peristaltic pumping of a third order fluid in a planar channel, Rheol. Acta 32, 47 - 56, 1993.

[15] T. Hayat, Y. Wang, A. M. Siddiqui, K. Hutter and S. Asghar, Peristaltic transport of a third order fluid in a circular cylindrical tube, Math. Mod. and Meth. in Appl. Sci. 12, 1691 - 1706, 2002.

[16] T. Hayat, Y. Wang, A. M. Siddiqui and K. Hutter, Peristaltic motion of a Johnson-Segalman fluid in a planar channel, Math. Prob. Eng. 1, $1-23,2003$.

[17] T. Hayat, Y. Wang, K. Hutter, S. Asghar and A. M. Siddiqui, Peristaltic transport of an Oldroyd-B fluid in a planar channel, Math. Prob. Eng. 4, $347-376$, 2004 .

[18] A. E. Hakeem, A. E. Naby and A. E. M. E. Misery, Effects of an endoscope and generalized Newtonian fluid on peristaltic motion, Appl. Math. Comput. 128, $19-35,2002$.

[19] R. A. Ranachandra and S. Usha, Peristaltic pumping in a circular tube in the prescence of an eccentric catheter, J. Biomech. Eng. 117, 448-454, 1995.

[20] R. Roos and P. S. Lykoudis, The fluid mechanics of the ureter with an inserted catheter, J. Fluid Mech. 46, 625-630, 1971.

[21] S. Yaniv, D. Elad, A. J. Jaffa and O. Eytan, Biofluid aspects of embryo transfer, Ann. Biomed. Eng. 31, 1255-1262, 2003.

[22] V. K. Sud, G. S. Sephon and R. K. Mishra, Pumping action on blood flow by a magnetic field, Bull. Math. Biol. 39, 385 - 390, 1977.

[23] H. L. Agrawal and B. Anwarudin, Peristaltic flow of blood in a branch, Ranchi Univ. Math. J. 15, 111 - 118, 1984. 
[24] Kh. S. Mekheimer, Peristaltic flow of blood under effect of a magnetic field in a non-uniform channels, Appl. Math. Comput. 153, 763-777, 2004.

[25] K. A. Helmy, Non-steady MHD flow for a non-Newtonian fluid with variable conductivity, Meccanica 30, 175-185, 1995.

[26] K. A. Helmy, MHD boundary layer equations for power-law fluids with variable electric conductivity, Meccanica 30, 187-200, 1995.

[27] N. Ali and T. Hayat, Peristaltic flow of a micropolar fluid in an asymmetric channel, Comput. Math. Applic. 55, 589-608, 2008.

[28] T. Hayat, A. Afsar, M. Khan and S. Asghar, Peristaltic transport of a third order fluid under the effect of a magnetic field, Comput. Math. Applic. 53, 1074-1087, 2007.

[29] T. Hayat, E. Momoniat and F. M. Mahomed, Effects of an endoscope and an electrically conducting third grade fluid on peristaltic motion, Int. J. Mod. Phys. B 22, 3997-4016, 2008.

[30] T. Hayat, E. Momoniat and F. M. Mahomed, Endoscope effects on MHD peristaltic flow of a power-law fluid, Math. Prob. Eng. 2006, 1-19, 2006.

[31] T. Hayat, N. Ahmad and N. Ali, Effects of an endoscope and magnetic field on the peristalsis involving Jeffrey fluid, Comm. Nonlin. Sci. and Num. Simul. 13, 1581-1591, 2008.

[32] N. Ali, T. Hayat and Y. Wang, MHD peristaltic flow of a third order fluid in an asymmetric channel, Int. J. Num. Meth. in Fluids doi: 10.1002/fld.2184, 2009 .

[33] N. Ali, Y. Wang, T. Hayat and M. Oberlack, Slip effects on the peristaltic flow of a third grade fluid in a circular cylindrical tube, J. Appl. Mech. 76, 011006-1-01006-10, 2009.

[34] T. Hayat, E. Momoniat and F. M. Mahomed, Peristaltic MHD flow of third grade fluid with an endoscope and variable viscosity, J. Nonlin. Math. Phys. 15, Suppl. 1, 91-104, 2008.

[35] S. Nadeem and S. Akram, Heat transfer in a peristaltic flow of MHD fluid with partial slip, Comm. in Nonlin. Sc. and Num. Simul. 15,

312-321, 2010. 
[36] S. Srinivas and M. Kothandapani, The influence of heat and mass transfer on MHD peristaltic flow through a porous space with compliant walls, Appl. Math. and Comput. 213, 197-208, 2009.

[37] A. Yildirim and S. A. Sezer, Effects of partial slip on the peristaltic flow of a MHD Newtonian fluid in an asymmetric channel, Math. Comput. Mod. doi:10.1016/j.mcm.2010.04.007.

[38] S. Nadeem and N. Sher Akbar, Influence of temperature dependent viscosity on peristaltic transport of a Newtonian fluid: Application of an endoscope, Appl. Math. and Comput. 10.1016/j.amc.2010.05.006.

[39] S. Nadeem and N. Sher Akbar, Numerical solutions of peristaltic flow of Williamson fluid with radially varying MHD in an endoscope, Int. J. Num. Meth. in Fluids, 10.1002/fld.2253.

[40] S. Nadeem and N. Sher Akbar, Exact and numerical simulation of peristaltic flow of a non-Newtonian fluid with inclined magnetic field in an endoscope, Int. J. Num. Meth. in Fluids, 10.1002/fld.2295. 